\title{
Setting, Clinical Pathways, Fast-Track and Rehabilitation Following Primary Knee Arthroplasty: A Literature Review
}

\author{
M.G. Benedetti ${ }^{*}, 1$, D. Sarti ${ }^{2}$, S. Bonfiglioli Stagni ${ }^{1}$ and E. Mariani ${ }^{1}$ \\ ${ }^{\text {I}}$ Physical Medicine and Rehabilitation Unit, Istituto Ortopedico Rizzoli, Bologna, Italy \\ ${ }^{2}$ Luce sul Mare Franchini Hospital, Santarcangelo di Romagna, Rimini, Italy
}

\begin{abstract}
In view of the high incidence of total knee replacements, it is important to identify the most efficient yet costeffective rehabilitation program, in order to optimize patient care within the constraints of the National Healthcare System.

This paper aimed at reviewing the literature on post-acute Total Knee Replacement (TKR) rehabilitation discharge setting, and on the strategies for early discharge of TKR patients from orthopedic wards.

Research studies on the effectiveness of rehabilitation in intensive rehabilitation care, skilled nursing facilities, home rehabilitation, fast-tracks, enhanced recovery and clinical pathways in total knee replacement patients have been reviewed.

Length of stay following knee arthroplasty is influenced by the following factors: age, sex, marital status, co-morbidity, preoperative use of walking aids, pre and postoperative hemoglobin levels, the need for blood transfusion, ASA (American Society of Anesthesiologists) score and time between surgery and mobilization.

Clinical pathways and fast tracks seem to be effective in reducing length of stay without increasing clinical complications. However, despite the vast quantity of literature available, results remain inconclusive. There is no clear evidence supporting an algorithm for the optimal rehabilitation management after total knee replacement.

Beside the question of which setting or path is preferable in terms of organization, the review enlightens that it is relevant also gaining a deeper understanding of the most important predictors of the best outcomes.

There is a need to review criteria for admitting total knee arthroplasty patients to intensive rehabilitation, to start a "fasttrack" protocol, to build "Clinical Pathways" and to discharge patients to home rehabilitation.
\end{abstract}

Keywords: Clinical pathways, fast track, rehabilitation, setting, total knee replacement.

\section{INTRODUCTION}

In Canada and United States more than 762.000 total knee arthroplasties were performed in 2010-2011 [1]. The increase in this type of operation is expected to increase markedly in the future $[2,3]$.

Together with the increase in the mean age of the population, the rate, predominance and diagnosis of knee arthritis have also increased [4]. Furthermore, because the lifespan of the prosthesis is longer (10-15 years) [2] several studies have highlighted how total knee replacement is increased among younger patients (between 50 and 60 years old) [5-7]. In a recent study, Weinstein et al. [8] have estimated that $4,007,400$ adults $(95 \%$ CI: $3,583,400$ to $4,431,400$ adults) in the United States over the age of fifty years currently live with a total knee replacement.

Post-acute rehabilitation has been recognized to be important to achieve optimal results [1]. However there are different views on rehabilitation delivery setting, in its intensity,

*Address correspondence to this author at the Physical Medicine and Rehabilitation Unit, Istituto Ortopedico Rizzoli, Via Pupilli 1, 40136 Bologna, Italy; Tel: +390516366236; Fax: +39051332392;

E-mail: benedetti@ior.it and in its duration [1]. Management and care of total knee replacement (TKR) patients varies within and across different health systems and variation exists in the process of care, not always evidence based. Financial reimbursement schemas often influence the management of TKR [9].

The socioeconomic burden of care for TKR rehabilitation is an emerging issue also in Italy and requires the adoption of proper measure [10].

Considering the increasing number of patients operated on TKR yearly, it is important to identify parameters that enable appropriate rehabilitation programs, to allocate correctly resources with respect to the patients needs avoiding disparities, and to contain the costs sustained by the National Healthcare System.

The present paper consists of a review of the literature on the setting proposed for post-acute TKR rehabilitation, and on the strategies used for early discharge from orthopaedic ward of patients operated on TKR.

\section{SETTING}

The primary post-acute rehabilitation care settings $[11,12]$ in United States are: Inpatient Rehabilitation 
Facilities (IRF), Skilled Nursing Facilities (SNF) and Home Health Agencies (HHA) $[12,13]$. The first means intensive hospital rehabilitation (almost three hours rehabilitation per day), the second indicates care in specialized centers (at least one hour rehabilitation), and the third refers to rehabilitation at home [12]. Of course there are differences among Health systems structures in different countries although this distinction can be generally accepted. Medical insurance, social-economic status, geographical distribution, and ethnic differences influence the choice treatment place [11].

Characteristics of patients with lower-extremity joint replacement treated in the three post-acute rehabilitation care settings vary [12]. In general, patients with lower functional independence are more likely to be admitted to IRFs than SNFs [13]. An early and intensive approach seems to provide a higher motor functional gain with respect to SNF, a better clinical outcome, and a higher patient's satisfaction in elderly people who live alone and with multiple painful comorbidities $[14,15]$. In other studies however IRF seems not to offer a significant benefit compared with SNF in terms of recovery of functional status [12]. Mahomed et al. [16] did not find differences in pain, functional outcome and patient's satisfaction between patients treated in intensive rehabilitation and patients who received home rehabilitation, but treatment at home was more convenient in terms of cost/benefit ratio. The direct home discharge has been found to be preferable in patients in good health and with active social support [12].

The literature is not univocal regarding the prevalence of comorbid conditions by type of post-acute rehabilitation care. DeJong [17] found that IRF patients had more comorbid conditions, whereas other authors found this to be the case for patients with joint replacement admitted to SNFs [12].

In a review performed in 2013, analyzing 101 patients undergoing knee replacement in 2009, Kathrins et al. [18] found that subjects discharged from Day Rehabilitation had similar or improved outcomes compared with subjects discharged from SNF at a lower cost and shorter stay. Results suggest that Day Rehabilitation delivered significant cost savings when compared with SNF without compromising patient outcomes. However when comparing outcome from different settings it should also be taken into account the amount of rehabilitation and health care received by patients after discharge from the orthoapedic ward [17], and the functional outcome in the long term after TKR, particularly in terms of knee function and patient's satisfaction [19].

With the aim of identifying the best candidates for inpatients rehabilitation after total joint arthroplasty, Vincent et al. [20-22] provided a series of research articles in which found that all patients admitted to an Intensive Rehabilitation Ward achieved good functional results but, to achieve a similar Functional Independence Measure (FIM) increase, a greater investment of time and money was required for revision arthroplasty. Patients in the $75 \%$ rule Centers for Medicare and Medicaid Services (CMS) criteria ( $>85$ years and obese Body Mass Index $>50$ ) have a slightly longer length of stay (LOS), reduced functional outcome and more difficulties on returning home.
However evidences in support of inpatient rehabilitation after total knee arthroplasty are still not consistent. A systematic review [23] revealed that there was no evidence to support the provision of inpatient rehabilitation after TKR over any outpatient-based alternative. Specifically, no randomized trial has compared inpatient rehabilitation to any group-based or center-based one-to-one program or a supervised or unsupervised home program. The authors reported that there was insufficient and inconsistent evidence to recommend any specific type, timing or setting for postacute TKR rehabilitation, and there was low therapeutic validity among trials related to exercise intensity, dosage and adherence. Longitudinal data from an Australian cohort [24] observed that no significant difference in health status at 12 months post-surgery was seen between patients discharged directly home and those admitted to intensive rehabilitation for knee replacement. As far as regards home rehabilitation outpatient, physical therapy performed in a clinic under the supervision of a trained physical therapist was found to provide the best long-term outcomes after surgery. When rehabilitation within an outpatient clinic is not feasible, unsupervised or remotely supervised therapy may be effective after TKR although the initial evidence suggests that telerehabilitation does not resolve range of motion, strength and functional impairments to the same extent as supervised physical therapy sessions including progressive exercise [25]. In a more recent clinical trial [26] no difference was found between patients performing supervised or standardized home program with respect to the effects on functional status concluding that a home exercise program can be used in the rehabilitation of patients with TKA, and implementation of home exercise programs can also reduce health-care spending.

Whilst the TKR procedure is viewed as highly cost effective in light of the impressive gains in functional performance and health-related quality of life, the acute care and associated rehabilitative costs impose a significant burden on public and private hospital budgets and resources $[24,27,28]$ such that cost effectiveness of TKR is reduced if the procedure is associated with a stay in an inpatient rehabilitation facility.

Based on these assumptions a randomized trial comparing the efficacy of the most resource-intensive form of rehabilitation delivery after TKR-inpatient rehabilitation compared to one with little resource use such as a monitored home program has been designed [23].

Early discharge to home, with home-based rehabilitation has been associated with reduced cost, improved clinical outcomes, and increased patient satisfaction [29]. Although home-based rehabilitation is increasingly seen as an essential component of the care path, it requires an adequate organization effort, patient and family engagement, shared decision-making, and great flexibility to accommodate changing needs. As advantage, therapy in the home environment gives the therapist the opportunity to identify and address the patient's unique needs in his or her own home speeding the resumption of Activities of Daily Living.

Artz et al. [30], in a UK survey, found that TKR patients reported a greater need for physiotherapy and seventy percent of the entire high volume orthopaedic centre refereed patients to outpatients physiotherapy. Targeted early 
rehabilitation at home resulted in reducing the length of hospital stay without an increase in complication rate with significant cost savings and without an increase in readmission [31]. A multidisciplinary team working on close collaboration was found to be the key to success of such a scheme.

The feasibility to provide discharge to home in the same day of operation was even explored by Berger et al. [32]. They found that nausea and vomit requiring treatment were the most common reasons to delay discharge, while no death, and no cardio-pulmonary complication were recorded.

\section{PREDICTING TOOLS FOR REHABILITATION NEEDS AFTER SURGERY}

The ability to predict discharge needs for hospital rehabilitation following TKR could assist caregivers, healthcare professionals and administrators in optimizing care and resource allocations for patients [33].

In 1998 Forrest et al. [34] investigated the predictive factors that might negatively influence the LOS in hospital and direct discharge from the orthopedic ward. Of the 125 patients considered, 101 were discharged directly whereas 24 were transferred to a rehabilitation unit. This study found that the need to transfer a patient to a rehabilitation ward was influenced by old age and diabetes as a combined disease.

In 2006, Bozic et al. [35] analyzed 7818 patients undergoing hip or knee arthroplasty, and found that predictive factors for hospital rehabilitation were advanced age ( $>80$ years), high ASA score, female gender and the presence of medical insurance.

Oldmeadow et al. [36] in 2003 developed the Risk Assessment and Prediction Tool (RAPT) to determine the expected need for hospital rehabilitation after surgery and before discharge from hospital. This tool uses six preoperative parameters (age, gender, ambulatory status, use of walking aids, community support (home help), and patient's choice to live with a caregiver following surgery) to identify the patient's potential need for intensive rehabilitation following TKR. The RAPT was shown to predict patient discharge to a rehabilitation facility following TKR with $89 \%$ accuracy for those with a high-risk score. Total RAPT score correctly predicted the discharge destination with $75 \%$ accuracy.

In a successive study Olmeadow et al. [37] observed that specific post-operative rehabilitation measured by RAPT increases the rate of direct discharged, thus reducing the length of stay in hospital after surgery.

Dauty et al. [38] used the RAPT to evaluate the risk of complications in patients hospitalized after TKR surgery. If the values were below 6 there was a high risk of the need for hospital rehabilitation; if the score was between 6 and 9 the risk was medium and if it exceeded 9 there was a good chance of discharging the patient directly.

The RAPT was used recently also among multi-ethnic patients undergoing TKR in Singapore [33] The findings suggest that RAPT items and scores, particularly the presence of a caregiver at home, and preferred discharge destination, can significantly predict the actual discharge destination and length of stay.

\section{STRATEGIES FOR EARLY DISCHARGE FROM THE ORTHOPEDIC WARD AFTER TKR}

After a TKR, the length of stay in a hospital or rehabilitation environment varies largely from a mean of 21 days (Japan), 12 days (Germany), 10 days (Italy), 9 days (UK), 3 days (USA) [39].

In general the length of stay is not only dependent on the clinical outcome, but is also influenced by logistical factors at the treatment center, the patient's clinical features, as well as traditions and cultural factors (urban or rural living environment) and personal factors (co-morbidities, social and marital status) [40]. Moreover, the national health reimbursement policies may also influence the length of stay after TKR.

\section{Clinical Pathways}

In 1980, clinical pathways have been implemented in the United States in an effort to reduce the length of the hospital stay and thereby control hospital costs, with less focus placed on consequences for patients and society [41].

Although evidences were provided that clinical pathways for knee replacement do not compromise safety and satisfaction of patients [42-44], their major impact is from a hospital perspective [41,45]. Protocols for clinical pathways coordinate in fact the activities of multifunctional teams (including physicians, nurses, physiotherapists) involved in providing care for patients with a particular diagnosis or required procedure [40].

The results of a meta-analysis [46] showed that clinical pathways could significantly improve the quality of care even if it was not possible to conclude that the implementation of clinical pathways is a cost-effective process, because none of the included studies analyzed the cost of the development and implementation of the pathways. Based on the results it was assumed that pathways have impact on the organization of care if the care process is structured in a standardized way, teams critically analyze the actual organization of the process, and the multidisciplinary team is highly involved in the re-organization. A strongly significant reduction in the length of stay after implementation of the clinical pathway was however observed, and even if it can be argued that a general trend towards a continuous reduction of length of stay has existed, in actual systems of care this was not observed in the control group.

However, to establish the cost-effectiveness of these interventions, Larsen et al. [41] suggested that they should be assessed also from a societal perspective.

In this respect Van Herck et al. [47] underlined how, despite the worldwide use of clinical pathways, it is unclear which key interventions multidisciplinary teams select as pathway components, which outcomes they measure, and what is the effect of this complex intervention. Joint arthroplasty clinical pathways address pre-admission 
education, pre-admission exercises, pre-admission assessment and testing, admission and surgical procedure, postoperative rehabilitation, minimal manipulation, symptoms management, thrombosis prophylaxis, discharge management, primary caregiver involvement, home-based physiotherapy and continuous follow-up. The impact of pathways for joint arthroplasty patients suggests that there is sufficient evidence that clinical pathways are an effective tool for improving process outcomes and financial outcomes. However, despite reported exceptions, and a research gap in thoroughly assessing effects on complications, there seems to be no effect on clinical outcome.

Krummenauer et al. [48] evaluated the effects of a clinical pathway for total knee arthroplasty in terms of patient- related (self-reported) daily life function and process cost profiles from the health care providers' perspective by means of a prospective pre-post cohort evaluation. In conclusion, the introduction of an interdisciplinary clinical pathway did not increase patient related outcomes in terms of the confirmatory outcome assessment (Western Ontario and McMaster Universities Osteoarthritis Index); on the contrary, during univariate confirmatory analysis the cost effectiveness from the health care providing Orthopedic Surgery Department's perspective was even found reduced. The authors concluded that, in terms of a cost-cost balance, the implementation of clinical pathways presents an investment into quality, but - at least after already having achieved a certain stage of process quality - may not necessarily result in cost reduction, but can even end up in increased human resource investment.

Analyzing possible innovative intervention to manage the rising costs and impending access problems facing orthopaedic patients, Mannings et al. [49] stated that the introduction of Integrated Care Pathways (ICPs) can offer a partial second- curve strategic solution. Both reduction of length of stay and clinical outcome improvements can be attributed to a better organization of care.

The Authors concluded that ICPs have lowered total joint replacement costs, complications, and hospital stay duration by facilitating interdisciplinary coordination and decreasing duplicative testing. Nevertheless, since providers are sometimes averse to changes in health-care delivery, implementation of ICPs may face resistance from orthopaedic surgeons, which needs to be overcome.

\section{Fast-Track/Accelerated/ Enhanced Recovery}

Based on the evidence that a successful TKR with a shorter length of stay is achievable, clinical pathways with fast-track approaches were introduced, firstly in Denmark [50] and become successively well established [51]. This consists of a multidisciplinary strategy involving patient education, multimodal analgesia, standardized perioperative anesthesia and local anesthetic infiltration, fluid administration, and early mobilization. It has been shown to reduce length of stay without increasing re-admission rates $[51,52]$. Other expedited or improved outcomes with fasttrack programs include functional rehabilitation and patient outcome [53] with increased satisfaction and safety after discharge [54]. Medical complications including thromboembolism are not more frequent [55] and mortality rate was reduced at two years following the introduction of a multimodal enhanced recovery protocol [56].

A prerequisite for the success of this approach is a multidisciplinary collaboration between patients, surgeons, anesthetists, physiotherapists, occupational therapists, although it was observed that whereas it can be cost-saving, no significant difference was found from a societal perspective [41].

However, to realize this, an effective and specific patient selection should be made by analyzing their clinical, social and demographic characteristics. Early discharge is closely correlated to the presence of dedicated professionals (nurses and physical therapists) who have a low rate of absenteeism, employing physical therapists with uniform case mixes, careful pre-surgery assessment, accurate patient selection according to age and comorbidity, effective communication and instructions given to patients and relatives, continuity of medical supervision, optimal pain therapy, early and aggressive physical therapy starting from the first day, and pre and postoperative spinal anesthesia with infiltration of local anesthetics [40].

According to Husted et al. [52], applying fast-track rehabilitation, discharge is possible within five days after surgery in $92 \%$ of cases and within three days in $41 \%$ of cases. Factors that might negatively influence discharge on the third day are advanced age, female gender, the patient living alone, the use of walking aids before surgery, pre and post operative hemoglobin levels, need for blood transfusion, the day of the week when the surgical procedure is performed, the time between surgery and mobilization and ASA score $\geq 3$. In a successive study Husted et al. [57] even found that all unselected TKR patients can be discharged within 3 days of surgery. Reduction of this time to 1-2 days may be achieved by improvement of perioperative analgesia (multimodal, non-opioid), reduction of the risk of orthostatic hypotension, improvement of quadriceps muscle function, and avoidance of logistical problems hindering early discharge.

Similar results were reported by Jorgensen and Kehlet [58]. Unselected fast-track TKR with a length of stay of $\leq 4$ days and discharge to home was feasible and safe, including in elderly patients with comorbidities.

They found also [59] that fast-track rehabilitation may also be applied to smokers and/or drinkers undergoing knee replacement and these risk factors do not influence the length of stay in hospital after surgery.

Also according to Schneider et al. [60] a successful fast track rehabilitation was possible without pre-selection and without compromising clinical safety in patients who underwent a fast track rehabilitation program within a groupdynamic set-up aiming for discharge day 3 to 5 postoperatively. However, a good social and physiotherapy community set-up should be available.

Raphael et al. [61] found that a multimodal multidisciplinary fast-track protocol reduced hospital stay and opioid consumption while maintaining a high level of patient safety. Program implementation is feasible both in tertiary care and in community hospitals. 
In the experience of Den Hertog et al. [62] the implementation of a 6 days pathway-controlled fast-track rehabilitation was achievable and beneficial when considering the American Knee Society Score (AKKS) and WOMAC scores, reduced intake of analgesic drugs, and reduced length of stay.

In a review of literature, Kehlet and Thienpont [63] summarized that length of stay after knee arthroplasty is influenced by preoperative risk factors, anaesthetic and surgical techniques, pain, orthostatic intolerance, cognitive function, sleep disturbances, bleeding and anaemia and finally muscle function and rehabilitation, and that fast-track surgery reduces the length of stay and the morbidity after knee arthroplasty.

\section{Factors Influencing Post-Op Patient's Recovery}

Several interventions can be adopted together to achieve better functional outcomes, enhance recovery, and reduce hospital stay. Non surgical intervention includes preoperative patients education to explain surgical procedure, symptoms management thus reducing their anxiety, preoperative nutritional status improvement, control of pain and optimization of muscular strength (i.e. electrostimulation of quadriceps), and pulsed electromagnetic field in the perioperative period to reduce inflammation and swelling [64].

Furthermore length of stay after knee arthroplasty is influenced by preoperative risk factors, anaesthetic and surgical techniques, pain, orthostatic intolerance, cognitive function, sleep disturbances, bleeding and anaemia and finally muscle function and rehabilitation [40].

Post-operative pain is a key factor for patient satisfaction; its treatment must be an integral part of the rehabilitation program [65]. The last 10 years have seen the use of intravenous therapy, femoral nerve block and epidural continuous infusion of analgesics for 24/48 hours with or without femoral nerve block. Since this system has side effects, multimodal protocols have been designed to prefer periarticular injections to parenteral drugs [66]. The most effective time to perform post-operative epidural analgesic for pain control is in the first 4-6 hours. The combination of a local anesthetic with a narcotic seems to be better than anesthetic alone [67]. The use of minimally-invasive surgical and computerized techniques reduces complications connected to surgery, due to a smaller surgical incision, faster and less painful rehabilitation, reduced LOS, faster resumption of daily life and a better ROM [68].

Ethnic, racial, and sexual factors, and the degree of articular damage are significantly negative especially for Afro-Americans, but generally for racial and ethnic minorities who already have a poorer preoperative score [69]. Usually women receive treatment in a more advanced stage of disease; therefore, more functional disability is present at the time of surgery. However, differences remain in the functional score also at subsequent follow-ups [70]. Older women had reduced functional gain, increased length of stay and more difficulties on returning home [71].
Concerning the rehabilitation of obese patients (BMI>30) inside the hospital, ROM and FIM were demonstrated to improve upon discharge independently of the type of arthroplasty (primary or revision). FIM is lower in revision surgery compared with primary arthroplasty and there are no differences between obese and not obese patients. The hospital costs for primary arthroplasty are lower than those of revision surgery, but they are directly correlated with BMI $[72,73]$.

In 2010 Stevens-Lapsley et al. [74] found that there were no significant differences between BMI and functional performance even in the sub-acute (1-3 months) and intermediate (6 months) period.

In summary, excessive BMI does not impede improvement during intensive rehabilitation, but this progress is less effective and is more costly in terms of physical and occupational therapy and pharmacotherapy [72]. Because female patients have a greater risk of functional limitation and "walking-aid dependence", specific intervention on the combined diseases and BMI in the preoperative period is necessary to improve post-operative function. In particular a study performed by Berend et al. [75] on patients over 89 years old, showed that the mean survival of patients treated surgically was comparable to that of the rest of the population and there was a significant improvement in the pain score and onset of perioperative medical complications only in $14 \%$ of cases. Therefore, after a careful patient selection, this operation is an excellent option even for the elderly.

According to the 2013 study by Jorgensen and Kehlet [58] the patient's age was correlated to a length of stay in hospital of more than 4 days after surgery especially if the patient was over 80 years old. In the age bracket of between 86 and 97 years old the mean LOS was 5 days. This is a novel finding respect to the 2011 study by Clement et al. [76], which reported a LOS of 8 days in patients between 80 and 92 years old undergoing THR or TKR.

Patients with post-operative hemoglobin $>10,5 \mathrm{~g} / \mathrm{dl}$ had reduced length of stay and improved FIM. This considered an indication for intensive rehabilitation. Those requiring blood transfusion had a longer length of stay, reduced exercise tolerance and reduced strength and functional status. However further studies are required to understand if optimized pre-operative hemoglobin is associated with reduced length of stay [77]. Patients admitted to rehabilitation have a potential to improve their motor function independently of their hemoglobin level. Nevertheless, reduced pre/post-operative levels of hemoglobin and FIM, old age, and hypertension, at the start of rehabilitation program, are risk factors for longer length of stay to achieve optimal rehabilitation objectives [78].

In 2012, Jans et al. [79] analyzed 5165 patients (662 already anemic preoperatively) undergoing THA or TKA (mean age: 67 years) and found that preoperative anemia was correlated to an increased possibility of receiving blood transfusions, returning to hospital within 90 days and a LOS of more than 5 days. 


\section{DISCUSSION}

In conclusion of the present review, notwithstanding the amount of existing literature, no clear evidence has been found for establishing an algorithm for the optimal rehabilitation management after TKR. According to Stinemann and Chan [80], it seems most important to look beyond the question of which setting is best, toward gaining a deeper understanding of the elements within these settings that most enhance outcomes.

There is a need to review some criteria to increase the appropriateness of admitting total knee arthroplasty patients to intensive rehabilitation, to start a "fast-track" protocol, to build "Clinical Pathways" and to discharge patients to home rehabilitation. Such strategies certainly require an organizational effort to create a continuum of care of the patients in the post-acute phase reducing costs without undermanage patients. But overall, besides costs assessment in terms of reduced length of stay, reliable clinical and functional outcomes should be identified in order to measure the actual effectiveness of the different rehabilitation setting. Probably the use of FIM to measure the appropriateness of an intensive rehabilitation should be integrated by other measures of comorbidities, rehabilitation complexity of patients at admittance, and functional outcome, disability and satisfaction in the long term.

Future studies should investigate and clarify which patients' characteristics factors may be more suitable for the choice of the rehabilitation setting.

\section{CONFLICT OF INTEREST}

The authors confirm that this article content has no conflict of interest.

\section{ACKNOWLEDGEMENTS}

Declared none.

\section{REFERENCES}

[1] Westby MD, Brittain A, Backman CL. Expert consensus on best practices for post-acute rehabilitation after total hip and knee arthroplasty: a Canada and United States Delphi study. Arthritis Care Res (Hoboken) 2014; 66(3): 411-23.

[2] Kurtz S, Ong K, Lau E, et al. Projections of primary and revision hip and knee arthroplasty in the United States from 2005 to 2030. J Bone Joint Surg Am 2007; 89(4): 780-5.

[3] Kurtz SM, Lau E, Ong K, et al. Future young patient demand for primary and revision joint replacement: national projections from 2010 to 2030. Clin Orthop Relat Res 2009; 467(10): 2606-12.

[4] Kozma CM, Slaton T, Paris A, Edgell ET. Cost and utilization of healthcare services for hip and knee replacement. J Med Econ 2013; 16(7): 888-96.

[5] Khatod M, Inacio M, Paxton EW, et al. Knee replacement: epidemiology, outcomes, and trends in Southern California: 17,080 replacements from 1995 through 2004. Acta Orthop 2008; 79(6): 812-9.

[6] Jain NB, Higgins LD, Ozumba D, et al. Trends in epidemiology of knee arthroplasty in the United States, 1990-2000. Arthritis Rheum 2005; 52(12): 3928-33.

[7] Mehrotra C, Remington PL, Naimi TS, et al. Trends in total knee replacement surgeries and implications for public health, 19902000. Public Health Rep 2005; 120(3): 278-82.
[8] Weinstein AM, Rome BN, Reichmann WM et al. Estimating the Burden of Total Knee Replacement in the United States. J Bone Joint Surg Am 2013; 95(5): 385-392.

[9] Lingard EA, Katz JN, Wright EA, Sledge CB. Predicting the outcome of total knee arthroplasty. J Bone Joint Surg Am 2004; 86A(10): 2179-86.

[10] Piscitelli P, Iolascon G, Di Tanna G, et al. Socioeconomic burden of total joint arthroplasty for symptomatic hip and knee osteoarthritis in the Italian population: A 5-year analysis based on hospitalization records. Arthritis Care Res (Hoboken) 2012; 64(9): 1320-7.

[11] Freburger JK, Holmes GM, Ku LJ, et al. Disparities in Post-Acute Rehabilitation Care for Joint Replacement. Arthritis Care Res 2011; 63(7): 1020-30.

[12] Mallinson TR, Bateman J, Tseng HY, et al. A Comparison of Discharge Functional Status After Rehabilitation in Skilled Nursing, Home Health, and Medical Rehabilitation Setting for Patients After Lower-Extremity Joint Replacement Surgery. Arch Phys Med Rehabil 2011; 92(5): 712-20.

[13] DeJong G, Horn SD, Smout RJ, et al. Joint Replacement Rehabilitation Outcomes on Discharged From Skilled Nursing Facilities and Inpatient Rehabilitation Facilities. Arch Phys Med Rehabil 2009; 90(8): 1284-96.

[14] DeJong G, Hsieh CH, Gassaway J, et al. Characterizing rehabilitation services for patients with knee and hip replacement in skilled nursing facilities and inpatient rehabilitation facilities. Arch Phys Med Rehabil 2009; 90: 1269-83.

[15] Walsh MB, Herbold J. Outcome After Rehabilitation for Total Joint Replacement at IRF and SNF: a case-controlled comparison. Am J Phys Med Rehabil 2006; 85(1): 1-5.

[16] Mahomed NN, Davis AM, Hawker G, et al. Inpatient compared with home-based rehabilitation following primary unilateral total hip or knee replacement: a randomized controlled. Trial J Bone Joint Surg Am 2008; 90(8): 1673-80.

[17] DeJong G, Tian W, Smout RJ, et al. Use of rehabilitation and other health care services by patients with joint replacement after discharge from skilled nursing and inpatient rehabilitation facilities. Arch Phys Med Rehabil 2009; 90: 1297-305.

[18] Kathrins B, Kathrins R, Marsico R, et al. Comparison of day rehabilitation to skilled nursing facility for the rehabilitation for total knee arthroplasty. Am J Phys Med Rehabil 2013; 92(1): 61-7.

[19] DeJong G, Tian W, Smout R, et al. Long-term outcomes of joint replacement rehabilitation patients discharged from skilled nursing and inpatient rehabilitation facilities. Arch Phys Med Rehabil 2009; 90: $1306-16$

[20] Vincent KR, Vincent HK, Lee LW, Alfano AP. Inpatient Rehabilitation Outcomes in Primary and Revision Total Knee Arthroplasty Patients. Clin Orthop Relat Res 2006; 446: 201-7.

[21] Vincent KR, Lee LW, Weng J, et al. A preliminary examination of the CMS eligibility criteria in total-joint arthroplasty. Am J Phys Med Rehabil 2006; 85(11): 872-81.

[22] Vincent KR, Vincent HK. A multicenter examination of the Center for Medicare Services eligibility criteria in total-joint arthroplasty. Am J Phys Med Rehabil 2008; 87(7): 573-84.

[23] Buhagiar MA, Naylor JM, Harris IA, et al. Hospital Inpatient versus HOme-based rehabilitation after knee arthroplasty (The HIHO study): study protocol for a randomized controlled trial. Trials 2013; 14: 432 .

[24] Tribe KL, Lapsley HM, Cross MJ, et al. Selection of patients for inpatient rehabilitation or direct home discharge following total joint replacement surgery: a comparison of health status and out-ofpocket expenditure of patients undergoing hip and knee arthroplasty for osteoarthritis. Chronic Illn 2005; 1(4): 289-302.

[25] Pozzi F1, Snyder-Mackler L, Zeni J. Physical exercise after knee arthroplasty: a systematic review of controlled trials. Eur J Phys Rehabil Med 2013; 49(6):877-92.

[26] Büker N, Akkaya S, Akkaya N, et al. Comparison of effects of supervised physiotherapy and a standardized home program on functional status in patients with total knee arthroplasty: a prospective study. J Phys Ther Sci 2014; 26(10):1531-6

[27] Zuckerman JD. Inpatient rehabilitation after total joint replacement. JAMA 1998; 279(11): 880.

[28] Lavernia CJ. Post discharge costs in arthroplasty surgery. J Arthroplasty 2006; 21(6 Suppl 2): 144-150. 
[29] Froimson MI. In-home care following total knee replacement. Cleve Clin J Med 2013; 80 (Suppl 1): eS15-8.

[30] Artz N, Dixon S, Wylde V, et al. Physiotherapy provision following discharge after total hip and total knee replacement: a survey of current practice at high-volume NHS hospitals in England and wales. Musculoskeletal Care 2013; 11(1): 31-8.

[31] Iyengar KP, Nadkarni JB, Ivanovic N, Mahale A. Targeted early rehabilitation at home after total hip and knee joint replacement: Does it work? Disabil Rehabil 2007; 29(6): 495-502.

[32] Berger RA, Kusuma SK, Sanders SA, et al. The feasibility and perioperative complications of outpatient knee arthroplasty. Clin Orthop Relat Res 2009; 467: 1443-9.

[33] Tan Ca, Loo G, Pua YH, et al. Predicting discharge outcomes after total knee replacement using the risk assessment and predictor tool. Physiotherapy 2014; 100(2): 176-81.

[34] Forrest G, Fuchs M, Gutierrez A, Girardy J. Factors Affecting length of stay and need for rehabilitation after hip and knee arthroplasty. J Arthroplasty 1998; 13(2): 186-90.

[35] Bozic KJ, Wagie A, Naessens JM, et al. Predictors of discharge to an inpatient extended care facility after total hip or knee arthroplasty. J Arthroplasty 2006; 21(6 Suppl 2): 151-6.

[36] Oldmeadow LB, McBurney H, Robertson VJ. Predicting Risk of Extended Inpatient Rehabilitation After Hip or Knee Arthroplasty. J Arthroplasty 2003; 18(6): 775-9.

[37] Oldmeadow LB, McBurney H, Robertson VJ, et al. Targeted postoperative care improves discharge outcome after hip or knee arthroplasty. Arch Phys Med Rehabil 2004; 85(9): 1424-7.

[38] Dauty M, Schmitt X, Menu P, et al. Using the Risk Assessment and Predictor Tool (RAPT) for patients after total knee replacement surgery. Ann Phys Rehabil Med 2012; 55(1): 4-15.

[39] Waddell J, Johnson K, Hein W, et al. Orthopedic Practice in Total Hip Arthroplasty and Total Knee Arthroplasty: Results From the Global Orthopaedic Registry (GLORY). Am J Orthop 2010; 39(9 Suppl): 5-13.

[40] Husted H. Fast-track hip and knee arthroplasty: clinical and organizational aspects. Acta Orthop Suppl 2012; 83(346): 1-39.

[41] Larsen K, Hansen TB, Thomsen PB, et al. Cost-effectiveness of accelerated perioperative care and rehabilitation after total hip and knee arthroplasty. J Bone Joint Surg Am 2009; 91(4): 761-72.

[42] Kim S, Losina E, Solomon DH, et al. Effectiveness of clinical pathways for total knee and total hip arthroplasty: literature review. J Arthroplasty 2003; 18(1): 69-74.

[43] Walter FL, Bass N, Bock G, Markel DC. Success of clinical pathways for total joint arthroplasty in a community hospital. Clin Orthop Relat Res 2007; 457: 133-7.

[44] Vanhaecht K, Bellemans J, De Witte K, et al. Does the organization of care processes affect outcomes in patients undergoing total joint replacement? J Eval Clin Pract 2010; 16(1): 121-8.

[45] Antrobus JD, Bryson GL. Enhanced recovery for arthroplasty: good for the patient or good for the hospital? Can J Anaesth 2011; 58(10): 891-96.

[46] Barbieri A, Vanhaecht K, Van HP, et al. Effects of clinical pathways in the joint replacement: a meta-analysis. BMC Med 2009; 7: 32.

[47] Van Herck P, Vanhaecht K, Deneckere S, et al. Key interventions and outcomes in joint arthroplasty clinical pathways: a systematic review. J Eval Clin Pract 2010; 16: 39-49.

[48] Krummenauer F, Guenther KP, Kirschner S. Cost effectiveness of total knee arthroplasty from a health care providers' perspective before and after introduction of an interdisciplinary clinical pathway--is investment always improvement? BMC Health Serv Res 2011; 11: 338.

[49] Manning BT, Callahan CD, Robinson BS, et al. Overcoming resistance to implementation of integrated care pathways in orthopaedics. J Bone Joint Surg Am 2013; 95(14): e100 1-6.

[50] Husted H, Jensen CM, Solgaard S, Kehlet H. Reduced length of stay following hip and knee arthroplasty in Denmark 2000-2009: from research to implementation. Arch Orthop Trauma Surg 2012; 132(1): 101-4.

[51] Malviya A, Martin K, Harper I, et al. Enhanced recovery program for hip and knee replacement reduces death rate. Acta Orthop 2011; 82(5): $577-81$
[52] Husted H, Holm G, Jacobsen S. Predictors of length of stay and patient satisfaction after hip and knee replacement surgery: fasttrack experience in 712 patients. Acta Orthop 2008; 79(2): 168-73.

[53] Larsen K, Hansen TB, Søballe K, Kehlet H. Patient-reported outcome after fast-track knee arthroplasty. Knee Surg Sports Traumatol Arthrosc 2012; 20(6): 1128-35.

[54] Kehlet H, Wilmore DW. Multimodal strategies to improve surgical outcome. Am J Surg 2002; 183(6): 630-41.

[55] McDonald DA, Siegmeth R, Deakin AH, et al. An enhanced recovery programme for primary total knee arthroplasty in the United Kingdom-follow up at one year. Knee 2012; 19(5): 525-9.

[56] Savaridas T, Serrano-Pedraza I, Khan SK, et al. Reduced mediumterm mortality following primary total hip and knee arthroplasty with an enhanced recovery program. A study of 4,500 consecutive procedures. Acta Orthop 2013; 84(1): 40-3.

[57] Husted $\mathrm{H}$, Lunn $\mathrm{TH}$, Troelsen A, et al. Why still in hospital after fast-track hip and knee arthroplasty? Acta Orthop 2011; 82(6): 67984.

[58] Jørgensen CC, Kehlet H. Role of patient characteristics for fasttrack hip and knee arthroplasty. Br J Anaesth 2013; 110(6): 972-80.

[59] Jørgensen CC, Kehlet H. Outcomes in smokers and alcohol users after fast-track hip and knee arthroplasty. Acta Anaesthesiol Scand 2013; 57(5): 631-8.

[60] Schneider M, Kawahara I, Ballantyne G, et al. Predictive factors influencing fast track rehabilitation following primary total hip and knee arthroplasty. Arch Orthop Trauma Surg 2009; 129(12): 158591.

[61] Raphael M, Jaeger M, van Vlymen J. Easily adoptable total joint arthroplasty program allows discharge home in two days. Can J Anaesth 2011; 58(10): 902-10.

[62] den Hertog A, Gliesche K, Timm J, et al. Pathway-controlled fasttrack rehabilitation after total knee arthroplasty: a randomized prospective clinical study evaluating the recovery pattern, drug consumption, and length of stay. Arch Orthop Trauma Surg 2012; 132(8): 1153-63.

[63] Kehlet H, Thienpont E. Fast-track knee arthroplasty -- status and future challenges. Knee 2013; 20(Suppl 1): 29-33.

[64] Ibrahim MS, Khan MA, Nizam I, Haddad FS. Peri-operative interventions producing better functional outcomes and enhanced recovery following total hip and knee arthroplasty: an evidencebased review. BMC Med 2013; 11: 37.

[65] Bergés IM, Ottenbacher KJ, Smith PM, et al. Perceived pain and satisfaction with medical rehabilitation after hospital discharge. Clin Rehabil 2006; 20(8): 724-30.

[66] Maheshwari AV, Blum YC, Shekhar L, et al. Multimodal pain management after total hip and knee arthroplasty at the Ranawat ortopaedic center. Clin Orthop Relat Res 2009; 467(6): 1418-23.

[67] Choi PT, Bhandari M, Scott J, Douketis J. Epidural analgesia for pain relief following hip or knee replacement. Cochrane Database Syst Rev 2003; (3): CD003071.

[68] Zanasi S. Innovations in total knee replacement: new trends in operative treatment and changes in peri-oprative management. Eur Orthop Traumatol 2011; 2(1-2): 21-31.

[69] Lavernia CJ, Alcerro JC, Contreras JS, Rossi MD. Ethnic and racial factors influencing well-being, perceived pain, and physical function after primary total joint arthroplasty. Clin Orthop Relat Res 2011; 469(7): 1838-45.

[70] Parsley BS, Bertolusso R, Harrington M, et al. Influence of gender on age of treatment with TKA and functional outcome. Clin Orthop Relat Res 2010; 468(7): 1759-64.

[71] Vincent KR, Vincent HK, Lee LW, Alfano AP. Outcomes in total knee arthroplasty patients after inpatient rehabilitation: influence of age and gender. Am J Phys Med Rehabil 2006; 85(6): 482-9.

[72] Vincent KR, Lee LW, Alfano AP. Effect of obesity on impatient rehabilitation outcomes following total knee arthroplasty. Clin Rehabil 2007; 21(2):182-90.

[73] Vincent HK, Vincent KR. Obesity and inpatient rehabilitation outcomes following knee arthroplasty: a multicenter study. Obesity (Silver Spring) 2008; 16(1): 130-6.

[74] Stevens-Lapsley JE, Petterson SC, Mizner RL, Snyder-Mackler L. Impact of body mass index on functional performance after total knee arthroplasty. J Arthrop 2010; 25(7): 1104-9. 
[75] Berend ME, Thong AE, Faris GW, et al. Total joint arthroplasty in the extremely elderly: hip and knee arthroplasty after entering the 89th year of life. J Arthrop 2003; 18(7): 817-21.

[76] Clement ND, MacDonald D, Howie CR, Biant LC. The outcome of primary total hip and knee arthroplasty in patients aged 80 years or more. J Bone Joint Surg Br 2011; 93(9): 1265-70.

[77] Diamond PT, Conaway MR, Mody SH, Bhirangi K. Influence of hemoglobin levels on inpatient rehabilitation outcomes after total knee arthroplasty. J Arthrop 2006; 21(5): 636-41.
[78] Wang X, Rintala DH, Garber SL, Henson HK. Association of hemoglobin levels, acute hemoglobin decrease, age, and comorbidities with rehabilitation outcomes after total knee replacement. Am J Phys Med Rehabil 2005; 84(6): 451-6.

[79] Jans O, Jørgensen C, Kehlet H, Johansson PI. Role of preoperative anemia for risk of transfusion and postoperative morbidity in fasttrack hip and knee arthroplasty. Transfusion 2014; 54(3): 717-26.

[80] Stineman MG, Chan L. Commentary on the comparative effectiveness of alternative settings for joint replacement rehabilitation. Arch Phys Med Rehabil 2009; 90(8): 1257-9.

(C) Benedetti et al.; Licensee Bentham Open.

This is an open access article licensed under the terms of the Creative Commons Attribution Non-Commercial License (http: //creativecommons.org/licenses/bync/3.0/) which permits unrestricted, non-commercial use, distribution and reproduction in any medium, provided the work is properly cited. 